\title{
Physiological Response of Mung Bean to Polyethylene Glycol Drought Stress at Flowering Period
}

\author{
Zhichao Yin ${ }^{*}$, Jie Liang², Xiyu Hao ${ }^{2}$, Huan Lu${ }^{1}$, Jianjun Hao1\#, Fengxiang Yin ${ }^{2 \#}$ \\ ${ }^{1}$ Biotechnology College, Shenyang Agricultural University, Shenyang, China \\ ${ }^{2}$ Baicheng Academy of Agricultural Sciences, Baicheng, China \\ Email: "Haojianjun106@126.com, "yinfx@163.com
}

Received 17 January 2015; accepted 25 March 2015; published 30 March 2015

Copyright (C) 2015 by authors and Scientific Research Publishing Inc.

This work is licensed under the Creative Commons Attribution International License (CC BY). http://creativecommons.org/licenses/by/4.0/

(c) (i) Open Access

\begin{abstract}
The study was carried out on the basis of the flowering period of mung bean (Phaseolus radiatus). The polyethylene glycol 6000 (PEG6000) was used to stimulate water stress. Four mung bean varieties with different drought resistances were used as materials in this experiment, in which the physiological indexes of mung bean for the identification of drought resistance were selected, and the response analysis of seven physiological indexes to different degrees of drought stress was measured by using polyethylene glycol 6000 (PEG6000) on different concentrations of 5\%, 10\% and $20 \%$. The physiological mechanism was analysed on drought tolerance of mung beans. This test was to set up identification system for drought tolerance of flowering mung bean. The results showed that the relative conductivity after 6 days of 5\% PEG treatment, the SOD activity after 6 days of 5\% PEG treatment, the POD activity after 6 days of 10\% PEG treatment and the ABA content after 6 days of 10\% PEG treatment could be used as drought-tolerant identification for mung beans at flowering period. These four physiological indexes and drought-resistant index weighted grey correlation analysis results showed that the correlation order was successively SOD activity $(0.8589)>A B A$ content $(0.8290)>$ conductivity $(0.7160)>$ POD activity $(0.6637)$; and the grey correlation degree was greater than 0.6500 among the four physiological indexes.
\end{abstract}

\section{Keywords}

Mung Bean, Flowering, Drought, PEG, Physiological Index

\footnotetext{
${ }^{*}$ First author.

${ }^{\#}$ Corresponding authors.
}

How to cite this paper: Yin, Z.C., Liang, J., Hao, X.Y., Lu, H., Hao, J.J. and Yin, F.X. (2015) Physiological Response of Mung Bean to Polyethylene Glycol Drought Stress at Flowering Period. American Journal of Plant Sciences, 6, 785-798. 


\section{Introduction}

Drought is one kind of environmental stresses which can cause considerable reductions in grain yield, especially during the reproductive stage. Around 30\% - 40\% decline in grain yield was documented under mid-drought stress, even without harvest under extremely drought stress [1]. The impact of drought stress on plant leads to cell convergence due to tissue dehydration, accompanied with cell elongation and other physiological processes blocked, cell wall hardening and programmed apoptosis accelerated. Furthermore drought impairs metabolism [2]; as a result, plants have stunted growth in addition to decreased chlorophyll content and photosynthesis, and the dry matter accumulation is inhibited [3].

Mung bean is a kind of traditional food of high nutritional and economic value. Also it has a certain function of detoxification, increasing appetite, and lowering blood pressure, cancer and other health effects [4]. Mung bean's growing environment has become increasingly barren, and drought is the major problem towards mung bean's growth. Therefore, cultivation of mung bean of drought-tolerant varieties plays a vital role in the future research. The physiological screening and the cultivar identification are establishing theoretical and practical foundation for drought-tolerant breeding of mung bean.

Plants have evolved complex mechanisms to perceive, respond and adapt to water deficit. For instance, they can avoid drought by maximizing water uptake, minimizing water loss or accumulating some osmolytes to cope with the stress. A lot of researches have done on the resistant physiological indexes in mung bean seedlings of open field and greenhouse [5]. The preliminary screening of seven indexes shows that Peroxidase (POD) activity, Superoxide Dismutase (SOD) activity and soluble sugar content, Abscisic acid (ABA) content, Malondialdehyde (MDA) content, superoxideanion $\left(\mathrm{O}^{2-}\right)$ production rate and relative electric conductivity (REC) can be used as identification for drought-tolerant mung beans [6].

The study is carried out on four mung bean varieties with polyethylene glycol (PEG) at the whole growing periods. This could help to have a better understanding of the physiological mechanisms of mung bean. Therefore it could provide a simple and fast way to the identification for drought tolerance of mung beans. This article is to deliver the research on the flowering stage; and the seedling and maturing parts will be delivered next.

\section{Materials and Methods}

\subsection{Plant Material}

Four mung bean varieties for the trial were selected from Institute of Edible Bean Crops, Baicheng City Academy of Agricultural Sciences, Baicheng City, Jilin Province, which included Bailv No. 9 (BL9), Bailv No. 11 (BL11), Dayinggelv 935 (BL935) and Dayinggelv (BL985). During the flowering and maturing stage, the functional leaves of the four mung bean varieties were picked up for measuring the physiological indexes for droughttolerance with three replications.

\subsection{Experimental Design}

The high quality seeds were sown in $1.5 \mathrm{~cm}$ depth of soil in the plastic bucket of $31 \mathrm{~cm} \times 22 \mathrm{~cm} \times 29 \mathrm{~cm}$, with cultivation density of 12 plants in each bucket under the outside condition. Water was irrigated timely until the three-leaf stage, $2000 \mathrm{ml}$ of PEG6000 solution were added for root dip of each culture; PEG was used to stimulate drought stress, and it was to set up four levels of processing, the first, water was added as control; the second, 5\% PEG water solution was added to the soil (mild drought); the third, 10\% PEG water solution were added to the soil (moderate drought); the fourth, 20\% PEG water solution were added to the soil (severe drought), with 3 replications. The functional leaves were picked up to determine the physiological indexes respectively in the third day, the sixth day, and the ninth day of the treatment.

\subsection{Test Methods}

Took DDS-IIA conductivity meter method to determine the relative conductivity, relative conductivity (\%) = (soaking the conductivity value boiling water bath conductivity values) 100\%; took anthronecolorimetry method to determine soluble sugars content; took thiobarbituric acid method to determine MDA content; took nitro blue tetrazolium (NBT) method to determine SOD activity; took guaiacol method to determine POD activity; took hydroxylamine method to determine $\mathrm{O}^{2-}$ production rate; took enzymes linked immunosorbent assay (ELISA) 
method to determine ABA content [7].

\subsection{Test Chemistry}

All chemistry were purchased form Shenyang Chemical Manufacture, the hormone assay kit was purchased from China Agricultural University.

\subsection{Statistical Analysis of Data}

Microsoft Office Excel 2003 was used to analyze the data and tabulation mapping, DPS data processing software was used for analysis of significant difference. The grey system theory modeling software (GTMS 3.0) was used to grey correlation analysis.

\section{Results and Analysis}

\subsection{Effects of PEG Drought Stress on Physiological Index of Flowering Mung Beans}

\subsubsection{Effects of PEG Drought Stress on Relative Conductivity of Flowering Mung Beans}

As shown in Figures 1(a)-(c), in mung bean flowering period after the mild and moderate drought stress of 5\% and 10\% PEG treatment, the conductivity change index of drought resistance varieties Bailv No. 9 showed a trend of rising with the increase of treatment days, after decline first; And it was showed a always keep a low level; the conductivity changes index of Bailv No. 11, Dayinggelv 935 and Dayinggelv 985 showed the trend of decline with the increase of treatment days, after first the rising and fall. Especially, the change of the differences was reached extremely significant level between each type, after 6th days of dealing with, the coefficient of variation was 0.3008 of maximum, and varieties of drought resistance performance, Bailv No. 9 and Bailv No. 11 of strong drought resistance varieties are relatively low, and Dayinggelv 935 and Dayinggelv 985 of weak drought resistance is relatively high.

Under 20\% PEG severe drought stress, the electrical conductivity change index of each type showed a trend of gradually rising decreased with the increase of treatment days, and drought-resistant varieties has been showing a low level. But, there is no significant change of the differences between each type, the variation coefficient was $0.1094-0.2358$. So, it should choose the conductivity after 6th days of 5\% PEG treatment as one of physiological indexes of drought resistance identification in flowering mung beans.

\subsubsection{Effects of PEG Drought Stress on $0^{2-}$ Production Rate of Flowering Mung Beans}

As shown in Figures 2(a)-(c), in flowering mung bean period after 5\% PEG treatment by water stress, different varieties of the ultraoxygen anion generating rate index with the increase of treatment days, showed a trend of decline after rising first; but the ultraoxygen anion producing rate of each type of index difference was not significant, the variation coefficient was 0.0335 - 0.0997. Under the condition of $10 \%$ and $20 \%$ PEG drought stress, the electrical conductivity of each type change index showed the trend of rise and fall, then change is not obvious difference among different varieties, the variation coefficient was $0.0178-0.1243$. So, the rate of $\mathrm{O}^{2-}$ produce cannot be used as one of identification indexes for drought-tolerance of flowering mung beans.

\subsubsection{Effects of PEG Drought Stress on the MDA Content of Flowering Mung Beans}

As shown in Figures 3(a)-(c), in the flowering period of mung beans after the mild and moderate drought stress of $5 \%$ and $10 \%$ PEG treatment, the change index of the MDA content of different varieties types with the increase of treatment days, all showed a trend of decline after rising first; But the performance was not significant difference among different varieties, the variation coefficient between $0.0784-0.1347$. Under severe drought stress of 20\% PEG treatment, the MDA content change index of Dayinggelv 985 with the increase of dealing with the number of days after the first increase gradually declining trend; the rest of the variety showed gradually rising trend with the increase of dealing with the number of days, however, the change of MDA content index differences between varieties was not significant, the variation coefficient between $0.0394-0.2185$, and the change rule do not agree with drought resistance of varieties. So, the MDA content of flowering period mung beans cannot serve as one of physiological indexes for the drought resistance identification.

\subsubsection{Effects of PEG Drought Stress on SOD Activity of Flowering Mung Beans}

As shown in Figures 4(a)-(c), in the flowering period of mung bean after different degree water stress of 5\%, 10\% 


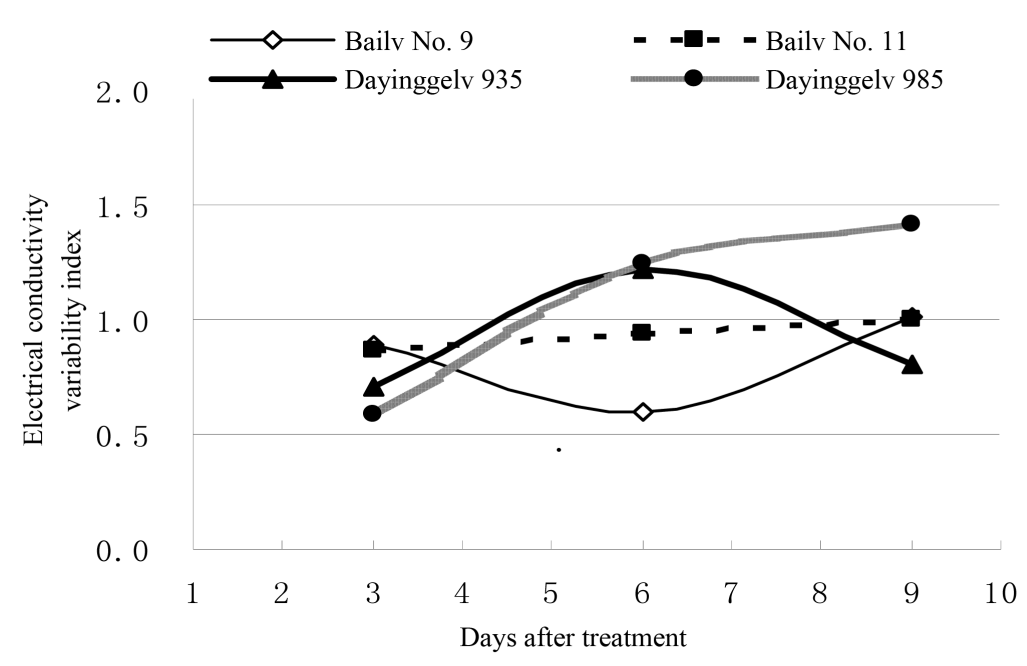

(a)

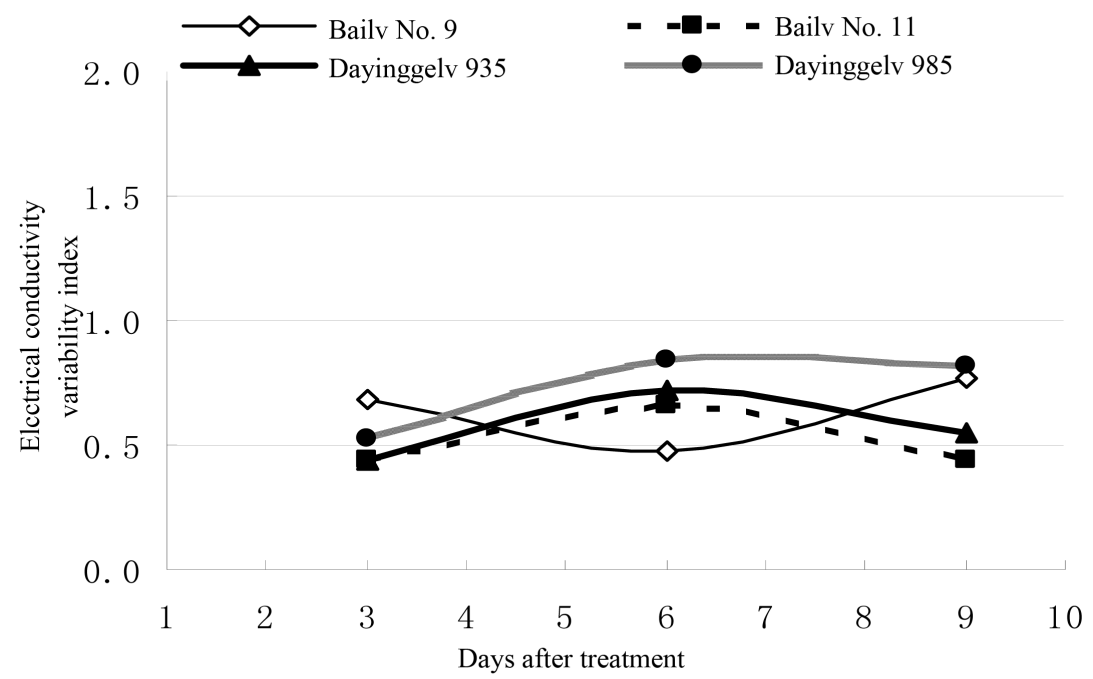

(b)

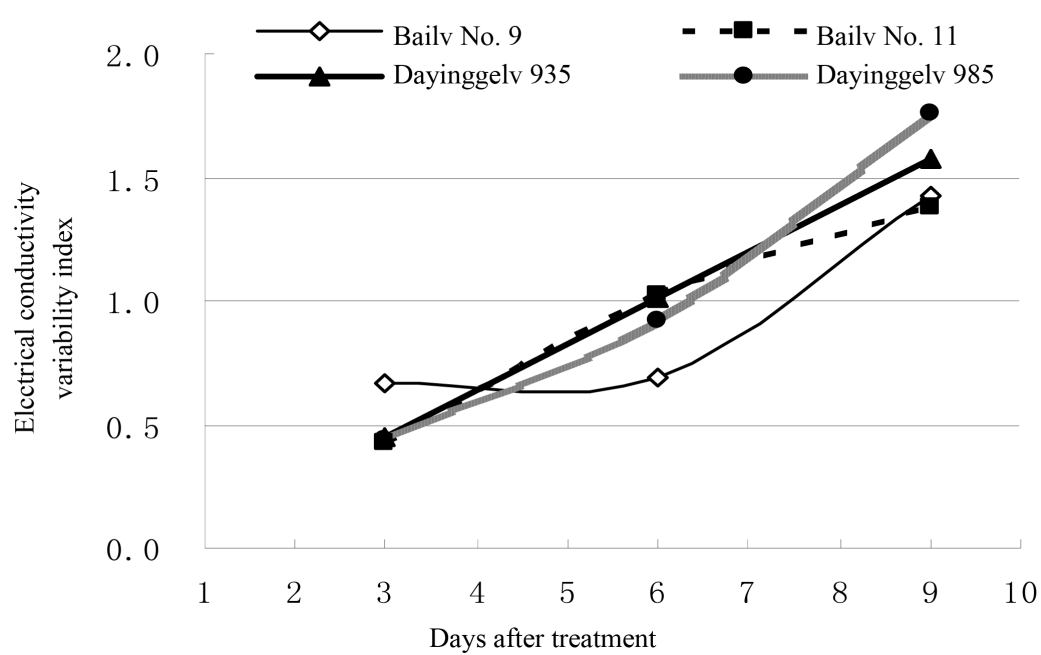

(c)

Figure 1. (a) Effects of 5\% PEC on electrical conductivity; (b) Effects of 10\% PEC on electrical conductivity; (c) Effects of 20\% PEC on electrical conductivity. 


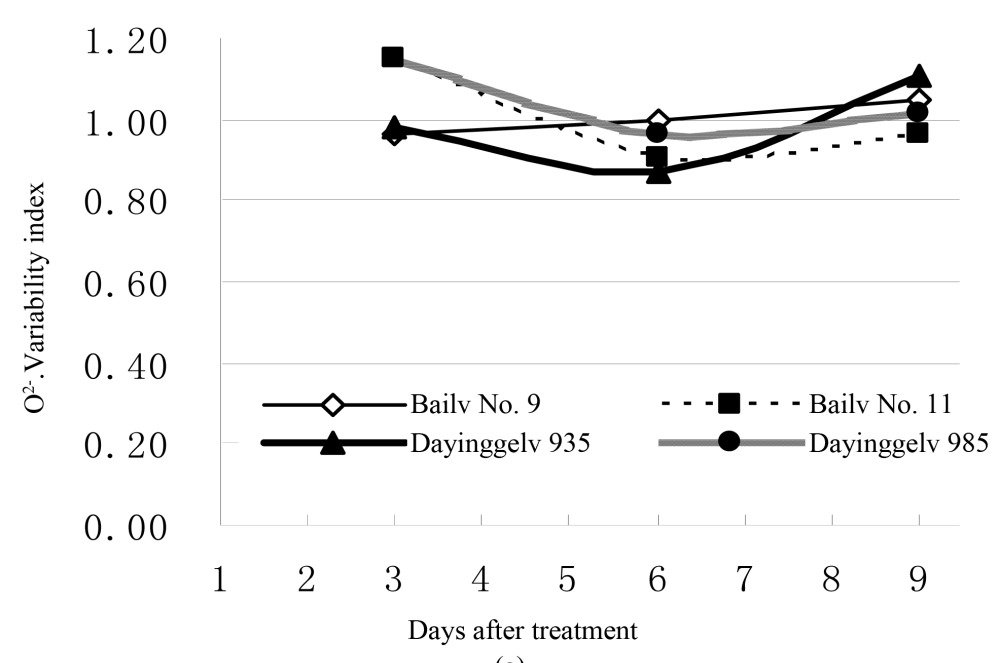

(a)

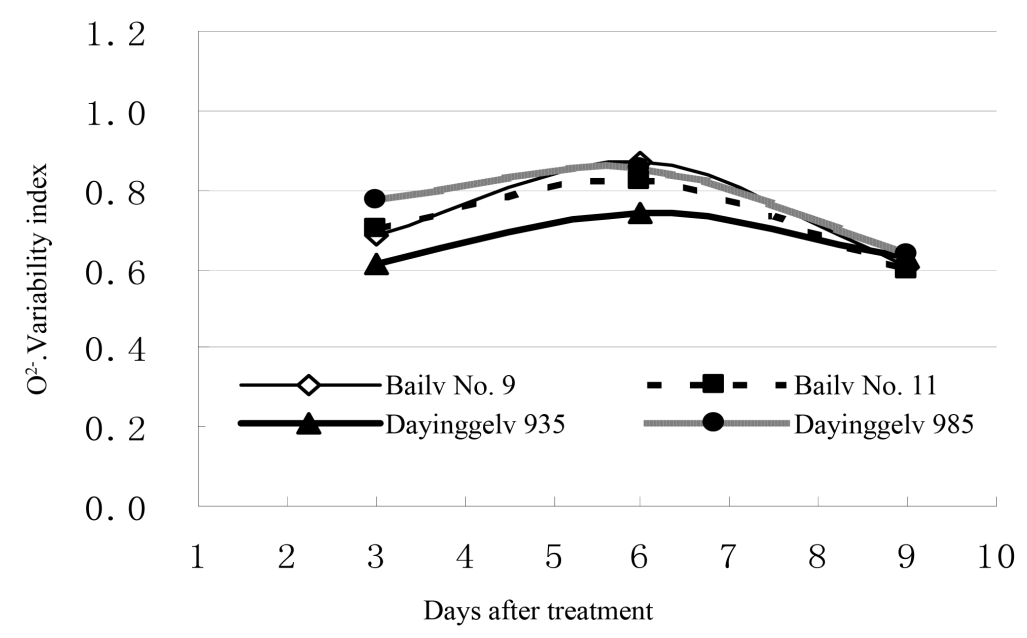

(b)

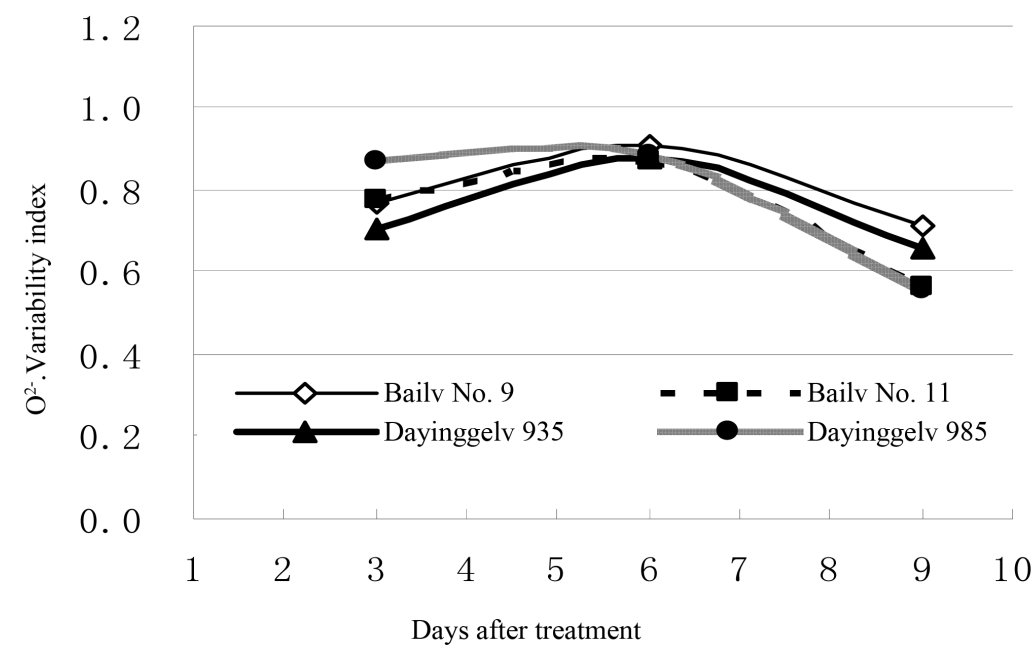

(c)

Figure 2. (a) Effects of 5\% $\mathrm{PEC}$ on $\mathrm{O}^{2-}$ product rate; (b) Effects of $10 \%$ PEC on $\mathrm{O}^{2-}$ product rate; (c) Effects of $20 \%$ PEC on $\mathrm{O}^{2-}$ product rate. 


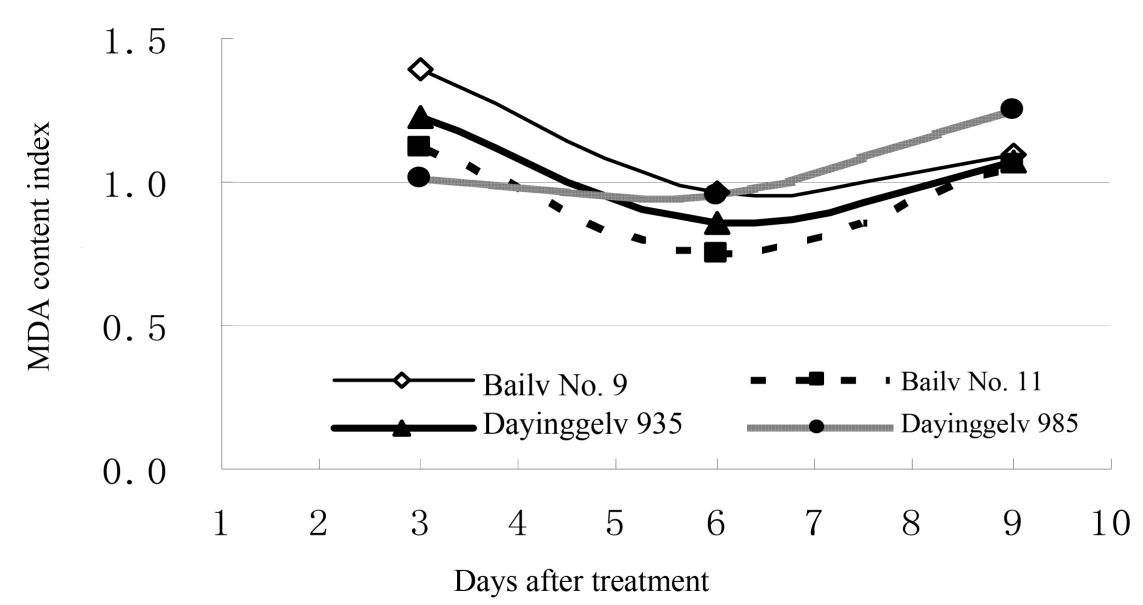

(a)

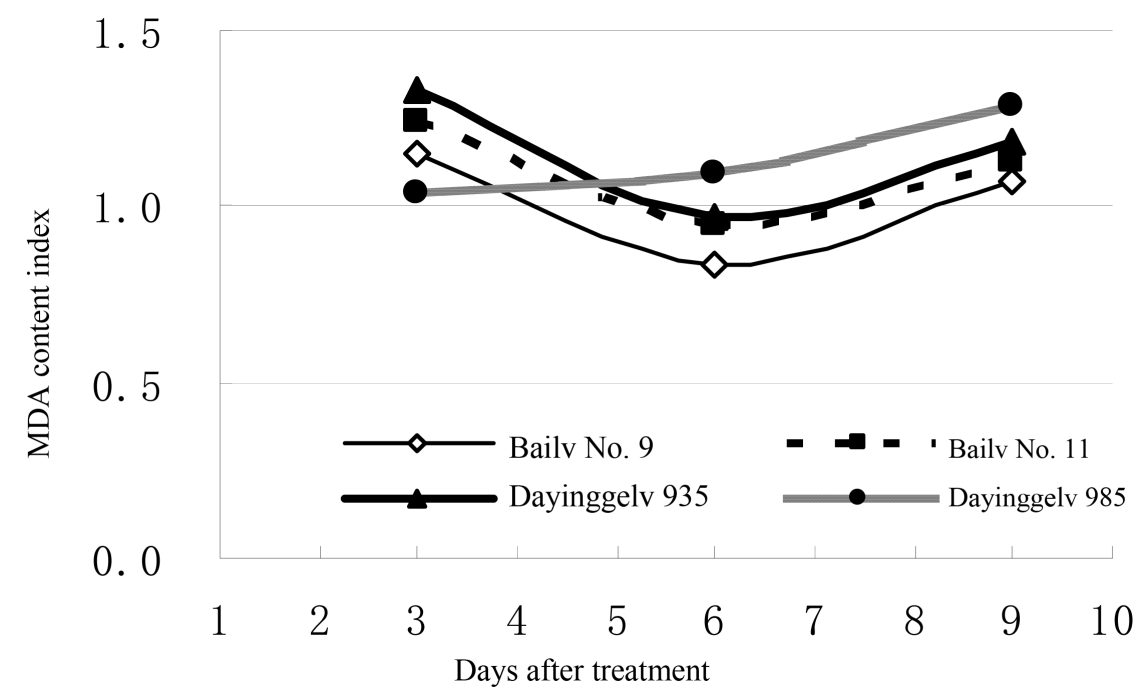

(b)

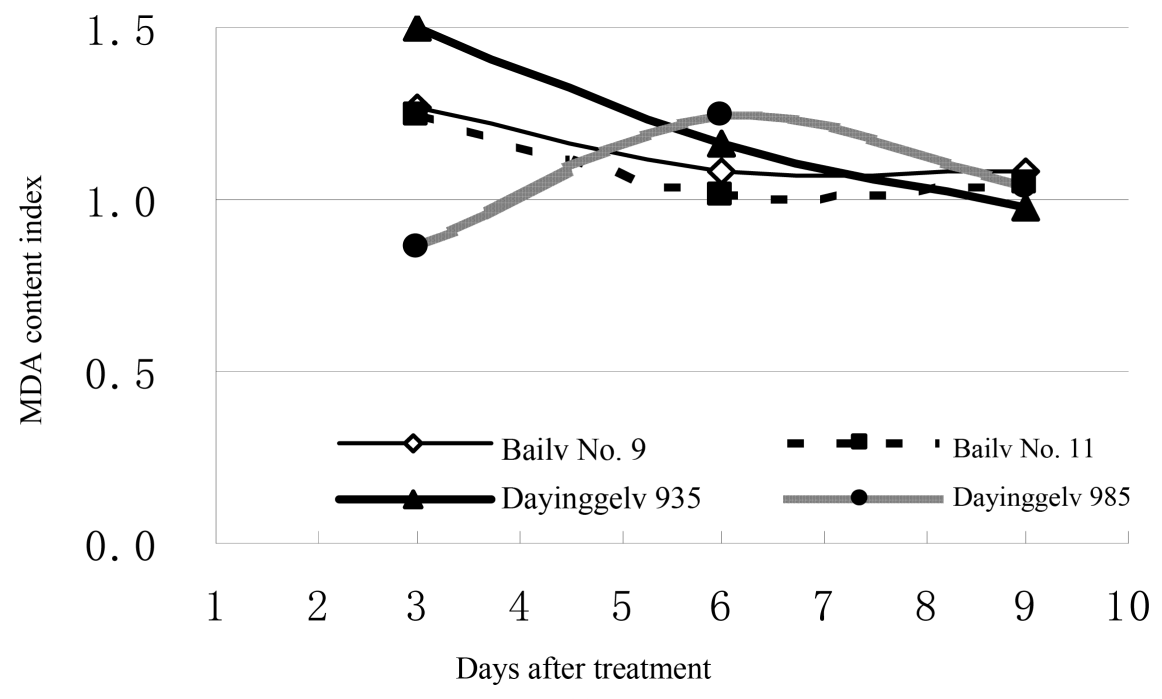

(c)

Figure 3. (a) Effects of 5\% PEC on the MDA content; (b) Effects of 10\% PEC on the MDA content; (c) Effects of $20 \%$ PEC on the MDA content. 


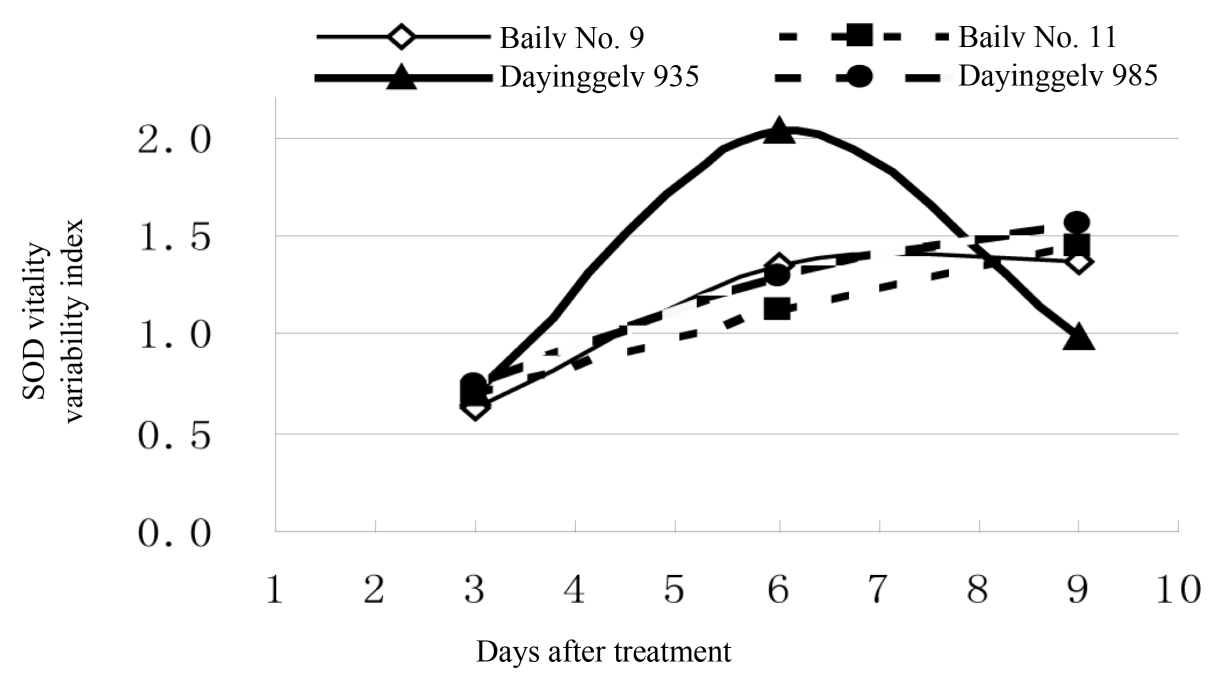

(a)

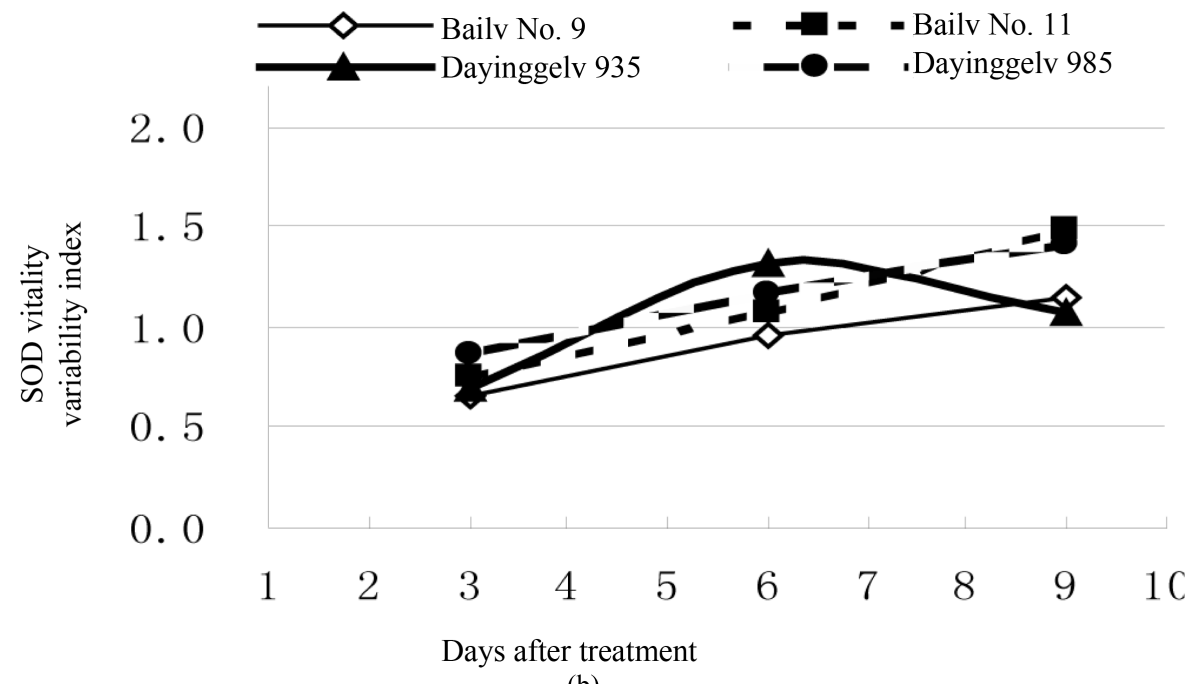

(b)

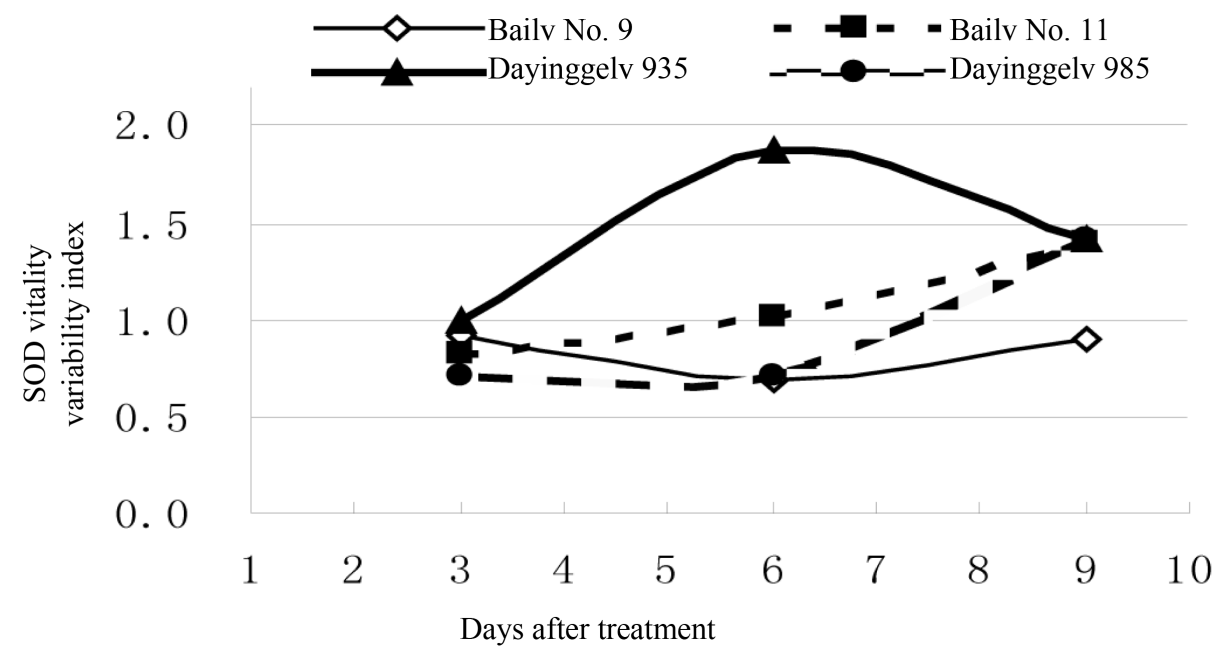

(c)

Figure 4. (a) Effects of 5\% PEC on SOD vitality; (b) Effects of 10\% PEC on SOD vitality; (c) Effects of $20 \%$ PEC on SOD vitality. 
and 20\% PEG treatment, the SOD activity change index of Dayinggelv 935 was showed the falling trend $\mathrm{n}$ after rising again with the increase of treatment days; after different concentration PEG treatment, the rest of the varieties was showed trend of increasing decreased with the increase of number of days. Especially it was showed changes extremely significant difference among different varieties after 6th days of 5\% PEG treatment by water stress, the variation coefficient were 0.2733; Dayinggle 935 and Bailv No. 9 was showed 2.0203 and 1.3467 of higher change index respectively; the changes index of Bailv No. 11 and Dayinggelv 985 is low, 1.1195 and 1.2969 respectively. After 6th days of 20\% PEG treatment by water stress, the SOD activity of change index was showed significant differences between the different varieties. But change law do not agree with drought resistance performance of varieties. So, It should choose the SOD activity as one of the physiological indexes for drought resistance identification after 6 days of 5\% PEG treatment in the flowering mung bean.

As shown in Figures 5(a)-(c), in the flowering period of mung bean after drought stress of 5\%, 10\% and 20\% PEG concentration processing, the POD activity change index of drought-resistant varieties Bailv No. 11 was showed a trend of gradually decline all after the rise first, with the increase of treatment days; and the POD activity changes index were higher after 6th days of PEG treatment of different concentrations, and it is change difference reached significant level with other varieties. The POD activity change index of other varieties was showed a trend of decreasing with increases of days after PEG treatment of various concentrations. After 6th days of $10 \%$ PEG treatment of moderate drought stress, especially changes of the POD activity was showed differences significantly between each varieties of type, the variation coefficient is 0.5272 . Generally consideration, the POD activity after 6th days of 10\% PEG treatment could be used as one of physiological identification indexes for drought resistance identification of flowering mung beans.

\subsubsection{Effects of PEG Drought Stress on ABA Content of Flowering Stage Mung Bean}

As shown in Figures 6(a)-(c), in the flowering period of mung beans after water stress of 5\%, 10\% and 20\% PEG treatment of different concentrations, the ABA content index of each varieties showed a trend of decline all after the rise, with the increase of treatment days; But in mild drought stress of 5\% PEG and severe drought stress conditions of $20 \%$ PEG. The change of the difference was not significant between each varieties. Above all, after 6th days of moderate drought stress of 10\% PEG treatment, the ABA content change was extremely significant difference between each varieties, the coefficient of variation was 0.6138 of maximum, and the change rule was consistent with drought resistance performance of varieties. So, the ABA content of 6th days of $10 \%$ PEG treatment could be used as one of the physiological indexes for drought resistance identification of mung beans at flowering stage.

\subsubsection{Effects of PEG Drought Stress on the Soluble Sugar Content of Flowering Mung Beans}

As shown in Figures 7(a)-(c), in the flowering period of mung bean after different concentrations of 5\%, $10 \%$ and 20\% PEG treatment, the soluble sugar content change index of leaves from each varieties was showed a trends of rise gradually all after the decline slightly, with the increase of treatment days. But all the soluble sugar content difference of each variety was not significant under the drought stress difference condition of different PEG. Only, the soluble sugar content of each varieties change was showed to reached level of extremely significant, after 9th days of $5 \%$ and $10 \%$ PEG treatment; the variation coefficient was 0.5021 and 0.4345 ; but change rule was not consistent with drought resistance performance of varieties. So, soluble sugar content can't be used physiological indexes for drought resistance identification of mung beans at flowering period.

\subsection{Grey Correlation Degree Analysis of Physiological Indexes for Drought Resistance Identification of Mung Bean Flowering Period}

It will filter out four physiological indexes of drought resistance identification of flowering mung beans that was the relative conductivity of 5\% PEG treatment on the sixth day, the SOD activity of 5\% PEG on the sixth day, the POD activity of 10\% PEG treatment on the sixth day, the ABA content of $10 \%$ PEG on the sixth day. And the change index and weighted drought index was summarized in Table 1. We were using the grey system theory modeling software (GTMS 3.0) for grey correlation analysis. And It will summarized grey correlation degree that received a physiological indexes by water stress of PEG drought for resistance identification at flowering period of mung beans and the drought index of weighted, and the correlation between the physiological indexes in Table 2. The results showed that the physiological indexes for drought resistance identification of anthesis of mung bean of PEG drought stress and weighted order of drought resistance index and relative size of grey 


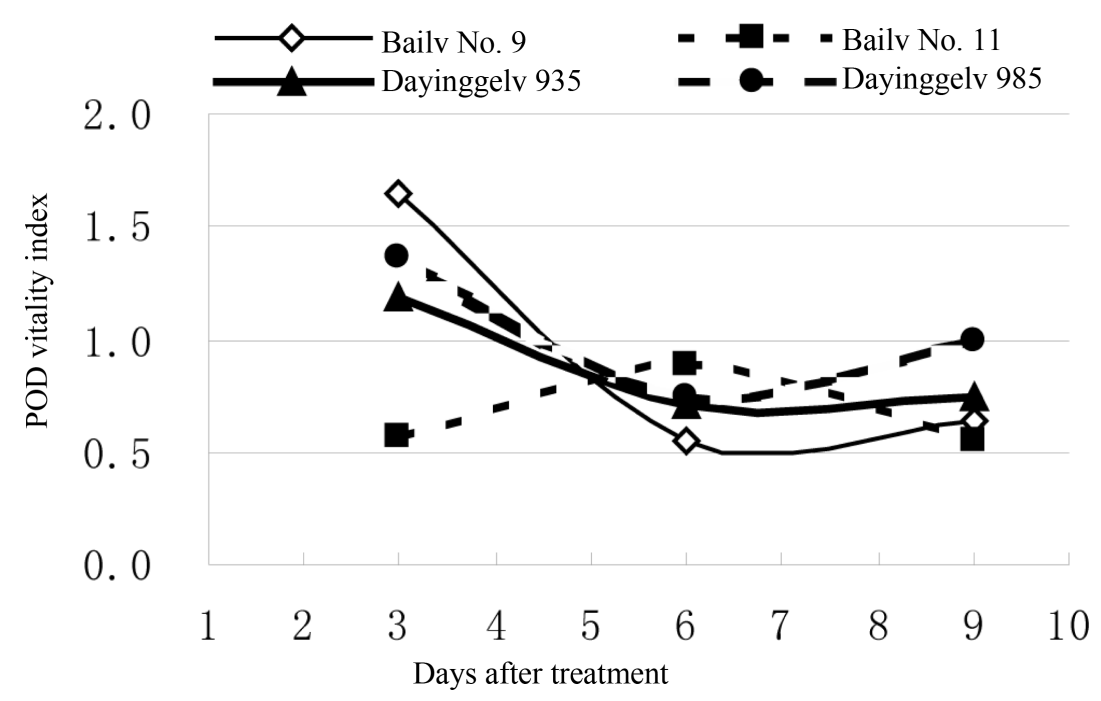

(a)

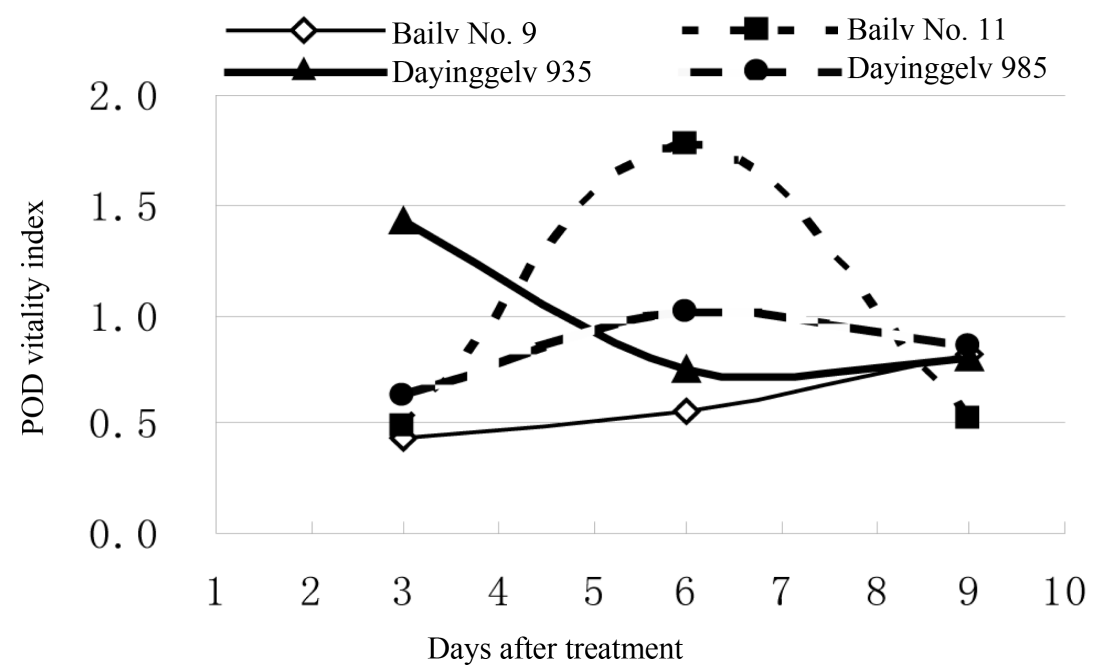

(b)

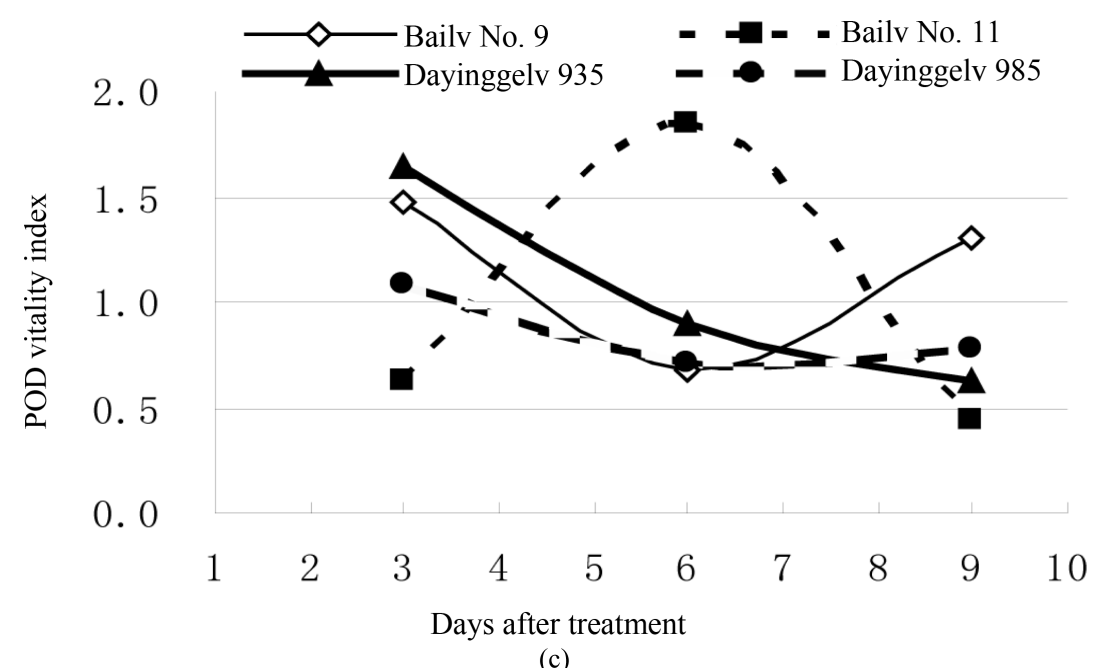

Figure 5. (a) Effects of 5\% PEC on the POD vitality; (b) Effects of 10\% PEC on the POD vitality; (c) Effects of $20 \%$ PEC on the POD vitality. 


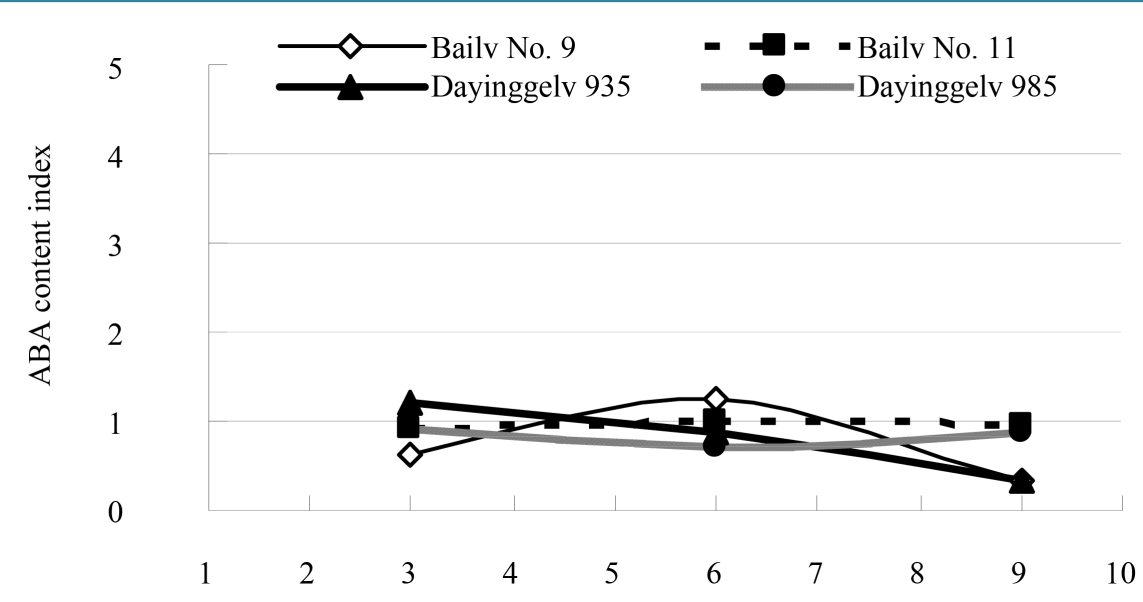

Days after treatment

(a)

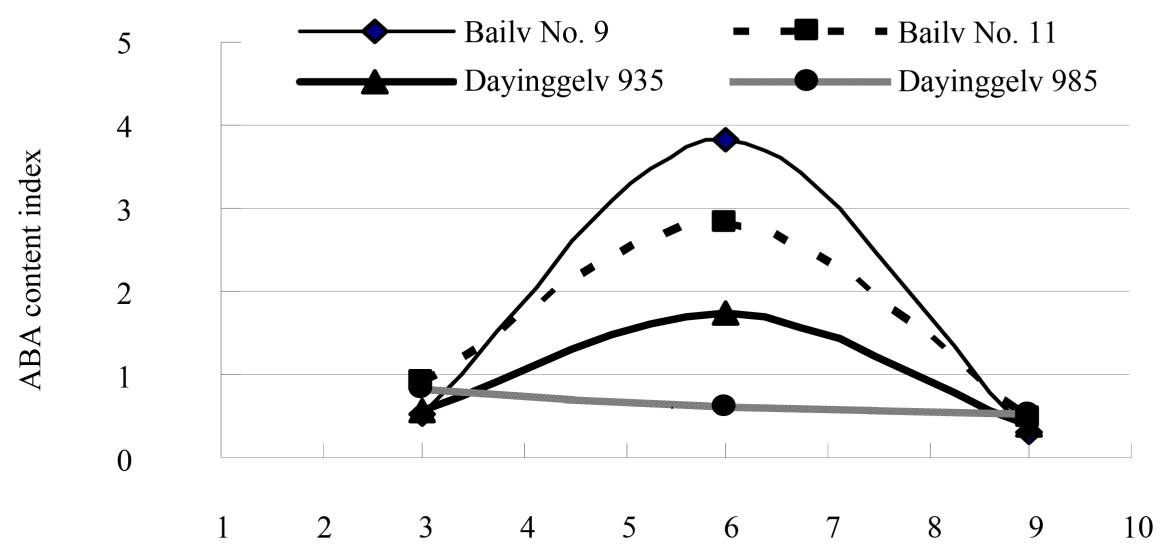

Days after treatment

(b)

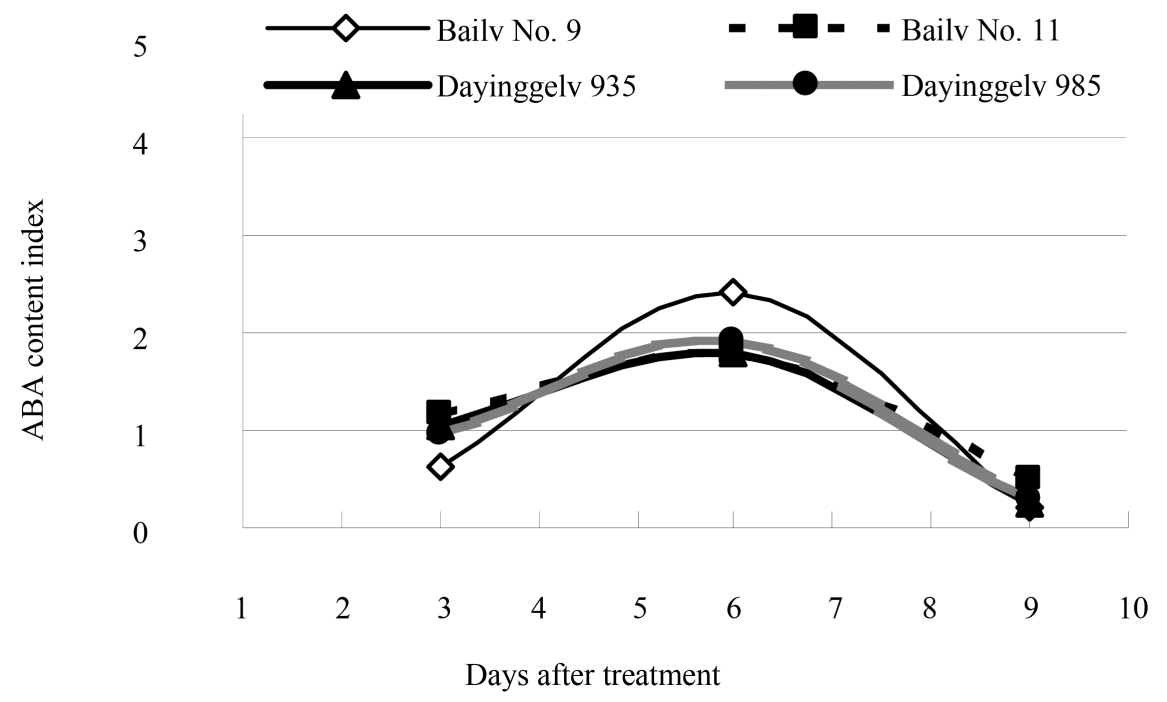

(c)

Figure 6. (a) Effects of 5\% PEC on the ABA content; (b) Effects of $10 \%$ PEC on the ABA content; (c) Effects of 20\% PEC on the ABA content. 


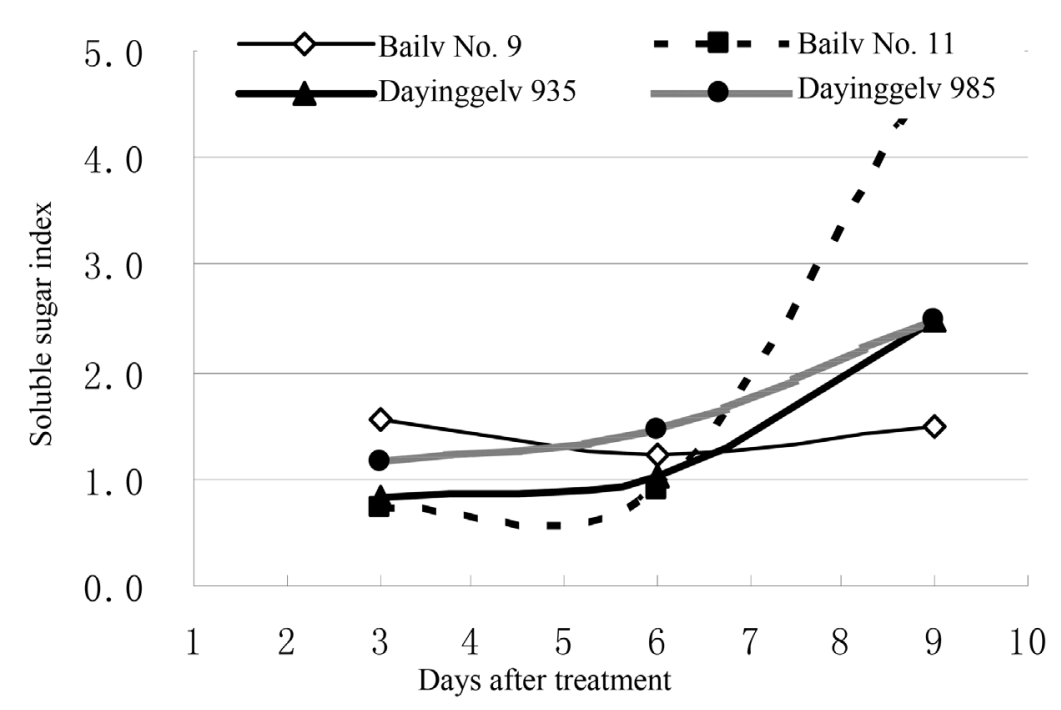

(a)

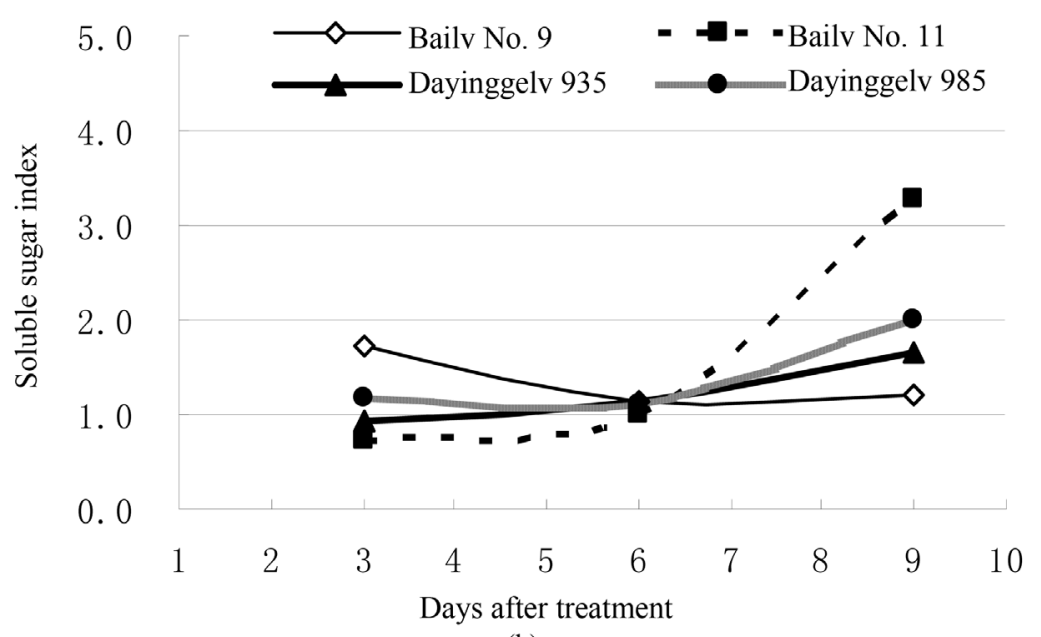

(b)

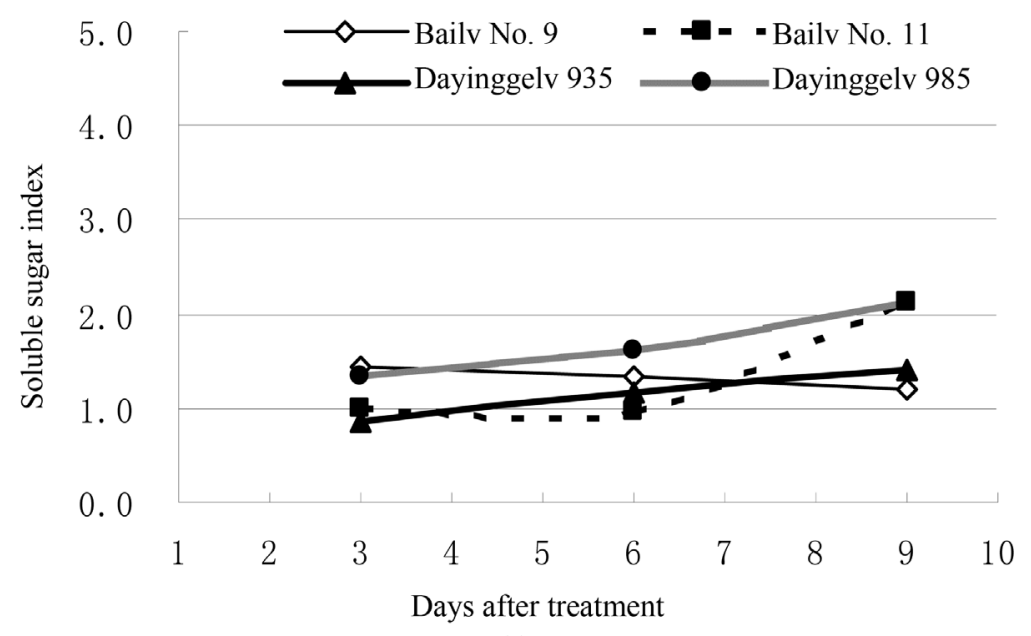

(c)

Figure 7. (a) Effects of 5\% PEC on the soluble sugar content; (b) Effects of 10\% PEC on the soluble sugar content; (c) Effects of 20\% PEC on the soluble sugar content. 
Table 1. Physiological changes the index of drought-resistant and weighted index of drought resistance under drought stress of PEG at flowering period of mung beans.

\begin{tabular}{cccccccc}
\hline Index & Bailv No. 9 & Bailv No. 11 & Dayinggelv 935 & Dayingglv 985 & Average & std & CV \\
\hline The weighted index & 6.114 & 5.7836 & 4.2993 & 2.6688 & 4.7164 & 1.5768 & 0.3343 \\
Conductivity of 5\% PEG & 0.6 & 0.9352 & 1.2139 & 1.2468 & 0.999 & 0.3005 & 0.3008 \\
SOD of 5\% PEG & 1.3467 & 1.1195 & 2.0203 & 1.2969 & 1.4458 & 0.3952 & 0.2733 \\
GPOD of 10\% PEG & 0.5544 & 1.7787 & 0.7419 & 1.0105 & 1.0214 & 0.5385 & 0.5272 \\
ABA of 10\% PEG & 3.8129 & 2.8206 & 1.7511 & 0.6082 & 2.2482 & 1.3799 & 0.6138 \\
\hline
\end{tabular}

Table 2. Physiology index of drought resistance grey relative relational grade analysis results under the condition of drought stress of PEG at bloom time of mung beans.

\begin{tabular}{cccccc}
\hline The grey correlation degree & The weighted index & $\begin{array}{c}\text { Conductivity of } \\
\text { 5\% PEG }\end{array}$ & $\begin{array}{c}\text { SOD of } \\
\text { 5\% PEG }\end{array}$ & $\begin{array}{c}\text { POD of } \\
\text { 10\% PEG }\end{array}$ & $\begin{array}{c}\text { ABA of } \\
10 \% \text { PEG }\end{array}$ \\
\hline The weighted index & 1 & 0.716 & 0.8589 & 0.6637 & 0.829 \\
Conductivity of 5\% PEG & 0.716 & 1 & 0.6651 & 0.879 & 0.8284 \\
SOD of 5\% PEG & 0.8589 & 0.6651 & 1 & 0.6175 & 0.7362 \\
POD of 10\% PEG & 0.6637 & 0.879 & 0.6175 & 1 & 0.7489 \\
ABA of 10\% PEG & 0.829 & 0.8284 & 0.7362 & 0.7489 & 1 \\
\hline
\end{tabular}

correlation degree was the SOD activity $(0.8589)>$ ABA content $(0.8290)>$ conductivity $(0.7160)>$ POD activity ( 0.6637$)$; and the grey correlation degree was greater than 0.6500 between the four physiological indexes.

\section{Discussion}

Effect of different treatments on $\mathrm{O}^{2-}$ levels in leaves of sugarcane seedlings, caused a significant increase in the levels of $\mathrm{O}^{2-}$ compared to the control, however, the increasing trends differed in different treatments [8]. The production rate of $\mathrm{O}^{2-}$ of rice flag leaf increased with the decrease of water potential. Under normal water condition, the $\mathrm{O}^{2-}$ production rate in transgenic rice was similar to that in the control, with no significant difference. Under drought stress, the $\mathrm{O}^{2-}$ production rate of the control increased fast [9].

The SOD activity increased slightly in all water-deficit treatments compared to control conditions at seedlings [10]. Drought stress caused little effect on SOD activity of rice anthers across the whole seedling stage [1].

Shoot and root POD activity in drought stressed garlic plants was found to increase significantly after 16th day of drought [11]. The highest POD activity under stress is due to stress treatment at the beginning of aggregation. The highest effect of drought stress on this enzyme activity is related to stress treatment at the beginning of aggregation [12]. POD in seedlings in drought treatments were higher than those in the check, the three former reached the highest on the 10th day after drought stress, and the latter was the highest on the 20th day after drought stress [13].

The role of plant hormone ABA in plants under drought stress is crucial in physiological responses that eventually lead to adaptation to an unfavorable environment [14]. The regulatory roles exerted by ABA deserve special interest. Indeed plant cells subjected to environmental conditions that disturb plant-water relationships leading to osmotic stress are well known to accumulate ABA [15]. ABA content increased within short time and reached a peak with prolonged time, when the seedlings were in severe water stress. The ABA level decreased quickly to the basal line within short time during rehydration. ABA content increased significantly in rice seedlings treated with exogenous ABA and PEG6000, and PEG-induced accumulation was effectively suppressed by these two inhibitors [16].

There was no significant difference in the contents of MDA between different genotypes of tomatoes under water stress. The MDA content was slightly decreased at first, and then was markedly increased at the 2nd and 3rd day after water cessation in all treatments [1]. The test results was showed that the MDA content of each mung bean varieties was presented the increase trend with increasing processing time of PEG drought stress, 
however, it was showed no significant changes compared with controls and the MDA content was not changed significantly between each PEG treatment, therefore the MDA content could not be used as identification index for drought-tolerance of mung bean seedlings.

The REC in the lower leaves increased progressively and was higher than in the upper leaves [17]. REC levels of rice varieties were significantly different under normal conditions or under drought stress were significantly than only under drought stress conditions, which may be mainly due to the better performance of in traits related to drought resistance. The values of traits on REC have been widely used as indices to evaluate drought resistance [18]. The REC was increased significantly for all the varieties after 9 days of 20\% PEG. It was demonstrated that the REC measuring of flowering mung beans should be extended the PEG treatment and the processing time, so the REC could not be used as identification index for drought-tolerance of flowering mung beans.

\section{Conclusions}

After analysis and discussion of this study, it could be determined that the relative conductivity after 6 days of $5 \%$ PEG treatment, the SOD activity after 6 days of 5\% PEG treatment, the POD activity after 6 days of 10\% PEG treatment and the ABA content after 6 days of 10\% PEG treatment could be used as drought-tolerant identification for mung beans at flowering period. The drought-tolerant strength of each variety of flowering mung bean could be identified based on the comprehensive analysis of these four physiological indexes and the comparison of testing results. These four physiological indexes and drought-resistant index weighted grey correlation analysis results showed that the correlation order was successively SOD activity $(0.8589)>$ ABA content $(0.8290)>$ conductivity $(0.7160)>$ POD activity (0.6637); and the grey correlation degree was greater than 0.6500 among the four physiological indexes.

About grey correlation analysis of the physiological indexes and morphological indexes for drought-resistant identification during the flowering stage of mung beans and about correlation analysis of the drought resistance identification indicators in different periods of mung bean, and the comparison of appraisal method has been needing to be in-depth studied.

\section{Acknowledgements}

This study was supported by Mung Bean Department, Baicheng Agriculture Academy of China, Biotechnology College and Shenyang Agricultural University and China Agriculture Research System (CARS-09-10). Here, for you to extend my deepest appreciation to the teacher's help and guidance.

\section{References}

[1] Fu, G.-F., Song, J., Xiong, J., Li, Y.-R., Chen, H.-Z., Le, M.-K. and Tao, L.-X. (2011) Changes of Oxidative Stress and Soluble Sugar in Anthers Involve in Rice Pollen Abortion under Drought Stress. Agricultural Sciences in China, 10, 1016-1025. http://dx.doi.org/10.1016/S1671-2927(11)60089-8

[2] Wan, F. (2006) World Overwintering Winter Wheat Improvement Theory and Practice. Agricultural Science and Technology Press, Beijing.

[3] Nielsen, D.C., Vigil, M.F. and Benjamin, J.G. (2009) The Variable Response of Dry Land Corn Yield to Soil Water Content at Planting. Agricultural Water Management, 96, 330-336. http://dx.doi.org/10.1016/j.agwat.2008.08.011

[4] Bouchenak, M. and Lamri-Senhadji, M. (2013) Nutritional Quality of Legumes, Their Role in Cardiometabolic Risk Prevention. Journal of Medicinal Food, 16, 185-198. http://dx.doi.org/10.1089/jmf.2011.0238

[5] Anjum, S.A., Farooq, M., Xie, X.-Y., Liu, X.-J. and Ijaz, M.F. (2012) Antioxidant Defense System and Proline Accumulation Enables Hot Pepper to Perform Better under Drought. Scientia Horticulturae, 140, 66-73. http://dx.doi.org/10.1016/j.scienta.2012.03.028

[6] Hao, J.J., Lu, W., Huang, C.H., Yu, Y. and Yin, F.X. (2013) Different Physicochemical Properties of Mung Bean Varieties Comparison. Agricultural Sciences-Jilin, China, 38, 19-21, 42.

[7] Hao, J.J. and Li, K.Z. (2005) Plant: Physiology Experimental Guide. Chemical Industry Press, China.

[8] Li, C.-N., Srivastava, M.-K., Nong, Q., Yang, L.-T. and Li, Y.-R. (2013) Molecular Cloning and Characterization of SoNCED, A Novel Gene Encoding 9-Cis-Epoxycarotenoid Dioxygenase from Sugarcane (Saccharum officinarum L.). Genes Genomics, 35, 101-109. http://dx.doi.org/10.1007/s13258-013-0065-9 
[9] Ding, Z.-S., Zhou, B.-Y., Sun, X.-F. and Zhao, M. (2012) High Light Tolerance Is Enhanced by Overexpressed PEPC in Rice under Drought Stress. Acta Agronomica Sinica, 38, 285-292. http://dx.doi.org/10.1016/S1875-2780(11)60106-5

[10] Silva, E.N., Ribeiro, R.V., Ferreira-Silva, S.L., Vieira, S.A., Ponte, L.F.A. and Silveira, J.A.G. (2012) Coordinate Changes in Photosynthesis, Sugar Accumulation and Antioxidative Enzymes Improve the Performance of Jatropha curcas Plants under Drought Stress. Biomass and Bioenergy, 45, 270-279. http://dx.doi.org/10.1016/j.biombioe.2012.06.009

[11] Borde, M., Dudhane, M. and Jite, P. (2012) Growth, Water Use Efficiency and Antioxidant Defense Responses of Mycorrhizal and Non Mycorrhizal Allium sativum L. under Drought Stress Condition. Annals of Plant Sciences, 1, 611.

[12] Ji, H., Fu, Q., Jiang, D.W., Shao, L. and Chen, L.Q. (2012) Physiological and Morphological Changes of Acer fabri Seedlings under Drought Stress. Acta Agriculturae Jiangxi, 24, 1-4.

[13] Poutari, S., Abbaspour, H., Sinaki, J.M. and Makarian, H. (2013) Studying Activities of Some Antioxidant Enzymes in Two Castor Grade under Drought Stress and Nitrogen Fertilizer. Bulletin of Environment, Pharmacology and Life Science, 2, 106-111.

[14] Zhang, L.X., Gao, M., Hu, J.J., Zhang, X.F., Wang, K. and Ashraf, M. (2012) Modulation Role of Abscisic Acid (ABA) on Growth, Water Relations and Glycinebetaine Metabolism in Two Maize (Zea mays L.) Cultivars under Drought Stress. International Journal of Molecular Sciences, 13, 3189-3202. http://dx.doi.org/10.3390/ijms13033189

[15] Larhera, F.R., Gagneulb, D., Niogreta, M-F., Emeryc, N., Monniera, C., Maerskalcka, V., Quilliena, G., Leforta, C., Plasmana, M., Boucharda, F. and Bouchereaua, A. (2013) Potentiation of Proline Accumulation in Oilseed Rape Leaf Discs Exogenously Supplied with Combinations of PEG and Cryoprotective Agents Is Associated with Overproduction of ABA. Environmental and Experimental Botany, 87, 167-178.

[16] Ye, N.H., Zhu, G.H., Liu, Y.G., Li, Y.X. and Zhang, J.H. (2011) ABA Controls $\mathrm{H}_{2} \mathrm{O}_{2}$ Accumulation through the Induction of OsCATB in Rice Leaves under Water Stress. Plant and Cell Physiology, 52, 689-698. http://dx.doi.org/10.1093/pcp/pcr028

[17] Zhang, W., Tian, Z.G., Pan, X.J., Zhao, X.M. and Wang, F. (2013) Oxidative Stress and Non-Enzymatic Antioxidants in Leaves of Three Edible Canna Cultivars under Drought Stress-Hort. Horticulture, Environment, and Biotechnology, 54, 1-8. http://dx.doi.org/10.1007/s13580-013-0070-6

[18] Ding, X.P., Li, X.K. and Xiong, L.Z. (2013) Insight into Differential Responses of Upland and Paddy Rice to Drought Stress by Comparative Expression Profiling Analysis. International Journal of Molecular Sciences, 14, 5214-5238. 